\title{
Modelo genérico de gestão da informação científica para instituições de pesquisa na perspectiva da comunicação científica e do acesso aberto
}

\author{
Fernando César Lima Leite \\ Sely Maria de Souza Costa *
}

Artículo recibido:

26 de agosto de 2014.

Artículo aceptado:

14 de mayo de 2015.
Resumo

Este artigo relata resultados de pesquisa que teve como objetivo geral propor modelo genérico de gestão da informação científica para instituições de pesquisa, tendo por base os fundamentos da comunicação científica e do acesso aberto. Trata-se de uma pesquisa de natureza teórica e exploratória. Do ponto de vista metodológico, é um estudo de abordagem mista que adotou a estratégia de triangulação concomitante. Os dados foram coletados por meio da aplicação de questionários, realização de entrevistas e lista de verificação e, em seguida, submetidos à análise estatística e de texto. Além da coleta e análise de dados empíricos, realizou-se análise de modelos de comunicação

* Ambos autores pertenecen a la Universidade de Brasília, Brasil. (fernandodfc@ gmail.com); (selmar@unb.br)

INVESTIGACIÓN BIBLIOTECOLÓGICA, Vol.30, Núm.69, mayo/agosto, 2016, México, ISSN: 0187-358X. pp. 43-73 
científica e de gestão da informação identificados na literatura. $\mathrm{O}$ universo da pesquisa foi constituído dos pesquisadores vinculados às unidades de pesquisa do Ministério da Ciência, Tecnologia e Inovação e a base amostral, definida a partir de amostragem não probalística intencional, foram os pesquisadores do Centro Brasileiro de Pesquisas Físicas e do Museu de Astronomia e Ciências Afins. Como principal resultado da investigação apresenta-se modelo genérico de gestão da informação científica para institutos de pesquisa, tendo por base a comunicação científica e o acesso aberto, em sua versão gráfica e textual. No modelo, fluxos de entrada e saída de informação são sistematizados por processos de gestão da informação científica que estão envolvidos pela perspectiva da comunicação científica e do acesso aberto. Sua estrutura, além de embutir conceitos essenciais, considera o ambiente do instituto de pesquisa como um sistema aberto onde ocorre o fluxo da informação científica. O modelo proposto é constituído também por elementos flexíveis que representam especificidades institucionais e disciplinares, e que variam em função dos contextos dos institutos de pesquisa. Além disso, todo o conjunto de elementos e relações entre eles estão sob influência constante de forças provenientes da comunidade científica em uma perspectiva ampla.

Palabras-chave: Comunicação Científica; Acesso Aberto; Gestão da Informação; Informação Científica; Comunicação na Ciência.

\section{Abstract}

A generic model of scientific information management for research institutes based on principles of scientific communication and open access

Fernando César Lima-Leite and Sely Maria de SouzaCosta

This paper presents theoretical and exploratory research whose aim is to propose a generic model of scientific information management for research institutes based on principles of scientific communication and open access. The mixed methodological approach adopts the concurrent triangulation strategy. Data collected using questionnaires, interviews and checklists 
were subjected to statistical and text analyses. In addition to collecting and analyzing empirical data, the study also presents an examination of the literature on communications and information management models. The object of study consisted of scientists associated with the Ministry of Science, Technology and Innovation; and the sample was acquired using intentional, non-probabilistic sampling of researchers of the Brazilian Centre for Physics Research and the Museum of Astronomy and Related Sciences. The study concludes with a generic model of scientific information management for research institutes built on the basis of scientific communication and open access to texts and graphical elements. In the model, the information input and output flows are systemized through scientific information management processes. Beyond imbedding keys, the structure approaches the research institute domain as an open system in which scientific information flows. The model proposed also offers flexible elements representing institutional and disciplinary particularities that vary depending on the context of each research institute. This set of elements and their interrelationships are, broadly speaking, under the constant influence of forces arising from the scientific community

Keywords: Scientific Communication; Open Access; Information Management; Scholarly Information; Scientific Information; Communication in Science.

\section{INTRODUÇÃO}

Tiante das realidades da informação digital em rede, da mutação do modo de produção do conhecimento e suas demandas diferenciadas de acesso, uso e disseminação da informação no ambiente científico, da necessidade de reestruturação do sistema de comunicação científica e subversão de sua lógica, instituições científicas estão imersas em um cenário de incerteza cujo ambiente informacional requer transformações concretas. Mais do que nunca, é necessário que as instituições sistematizem processos que otimizem o ciclo da informação que alimenta e que resulta das atividades de pesquisa. As práticas e metodologias de gestão da informação científica pautadas na 
estrutura do modelo tradicional de comunicação da ciência já não são suficientes para atender a estas expectativas.

A implementação de processos sistemáticos de gestão da informação científica norteados pelas demandas do novo ambiente informacional, promovem e otimizam fluxos de informação que são suportados pelo sistema de comunicação científica, de maneira que suas funções sejam potencializadas e ampliadas. A gestão da informação científica deve, portanto, considerar a nova perspectiva informacional e comunicacional do sistema científico. A comunicação da informação científica é um processo complexo que envolve e está presente ao longo de toda a cadeia de produção do conhecimento, ou seja, desde o momento em que pesquisadores formulam o problema de pesquisa até o momento do uso do novo conhecimento produzido por parte de outros pesquisadores. Assim, quanto mais apropriados ao contexto forem os processos de gestão da informação científica, mais coerentes e fluidos serão o processos de comunicação científica.

Desse modo, processos de gestão da informação científica devem corresponder às expectativas e comportamentos dos atores envolvidos e, ao mesmo tempo, serem compatíveis com a natureza da informação e do conhecimento científico e de sua produção e, naturalmente, com as forças e propriedades que governam e influenciam o seu fluxo. Desta feita, toda e qualquer influência sofrida pelo sistema de comunicação científica deve ser refletida nas práticas de gestão da informação científica.

Desde o surgimento da Internet, o maior evento que transformou, e não apenas modernizou, as bases sobre as quais o sistema de comunicação científica se estruturou foi a emergência de um movimento mundialmente conhecido como Acesso Aberto à Informação Científica. Na realidade, o acesso aberto constitui uma reação da comunidade científica à lógica do sistema de comunicação tradicional de comunicação da ciência, especialmente ao sistema de publicações. Seus pressupostos e estratégias compatibilizam esforços que contribuem para reestruturar/reformar o sistema de comunicação científica de modo que sejam removidas as barreiras presentes no fluxo da informação científica, como aquelas relacionadas com tecnologias, custos e direitos autorais. A principal intenção é fazer com que resultados de pesquisa científica estejam pública e permanentemente acessíveis e sem custo a quem possa interessar. Aliado a isso, por ser constituído também de processos de comunicação científica mais flexíveis, o modelo permite maior vazão a demandas das novas de formas de produção, compartilhamento e uso do conhecimento científico

A abordagem do acesso aberto tem se instituído gradativamente como modelo alternativo de comunicação da ciência. Por promoverem as condi- 
ções que favorecem um maior controle institucional da informação científica, as estratégias do acesso aberto, como uma expressão emergente de um novo cenário da comunicação científica, torna-se aspecto fundamental a ser considerado em iniciativas de gestão da informação científica em nível individual, institucional, nacional e internacional. Os resultados aqui relatados têm origem em pesquisa que teve como objetivo geral propor modelo genérico de gestão da informação científica para instituições de pesquisa, tendo por base os fundamentos da comunicação científica e do acesso aberto. O modelo genérico foi proposto a partir da i) identificação e descrição de modelos de gestão da informação e de comunicação científica, incluindo seus elementos e processos, da ii) identificação da percepção de pesquisadores, características das atividades de produção do conhecimento científico e do iii) mapeamento das atividades de busca, acesso e uso da informação, assim como hábitos de comunicação científica de pesquisadores de institutos de pesquisa.

\section{RELAÇÕES ENTRE GESTÃO DA INFORMAÇÃO CIENTÍFICA, COMUNICAÇÃO CIENTÍFICA E ACESSO ABERTO: UMA PERSPECTIVA CONCEITUAL}

Tendo em vista o objetivo geral da pesquisa, que foi propor modelo genérico de gestão da informação científica para instituições de pesquisa tendo por base os fundamentos da comunicação científica e do acesso aberto, foi realizada uma análise da literatura. A fundamentação teórica construída a partir da literatura permitiu explicitar as relações conceituais entre gestão da informação científica, comunicação científica e acesso aberto. Tais relações são apresentados a seguir como plataforma teórica sobre a qual o desenvolveu o estudo.

A primeira parte da construção teórica formulada evidencia as relações mais amplas existentes entre gestão da informação científica, comunicação científica e acesso aberto. Nesse sentido, a partir de diferentes perspectivas acerca do entendimento do que constitui a gestão da informação (Choo, 1998; Davenport, 1998; Detlor, 2009; Fairer-Wessels, 1997; Jaeger et al., 2005; Middleton, 2002; White, 1985; Wilson, 2002) a gestão da informação científica foi definida como o conjunto de políticas e processos que sistematizam a identificação de necessidades, coleta/aquisição, organização, armazenamento e preservação, recuperação, disseminação e uso da informação científica no contexto das instituições que a produzem. Levando em consideração os níveis de gestão da informação propostos por Rowley (1998) - sobretudo aquele que define os contextos informacionais, assim como o funcionamento do sistema de comunicação científica e de seus processos (Hills, 1983; Hurd, 1996, 2000, 2004; Shearer e Birdsall, 2002) foi possível definir a finali- 
dade da gestão da informação científica no âmbito de instituições de pesquisa. Tal finalidade diz respeito a promoção de condições para que a informação que alimenta e que resulta das atividades de pesquisa esteja disponível e acessível para que pesquisadores, dentro ou fora da instituição, gerem novos conhecimentos e, consequentemente, contribuam para o avanço da ciência.

Das considerações feitas resultou o entendimento de que para que a gestão da informação científica ocorra de modo apropriado, é necessário que seja levada em consideração uma série de peculiaridades do ambiente que envolve as comunidades científicas, sobretudo, aquelas que impactam, em qualquer medida, o fluxo da informação científica. Ou seja, as forças que influenciam o sistema de comunicação científica (Borgman, 2007), por impactarem o fluxo da informação, influenciam, do mesmo modo, os processos de gestão da informação (Choo, 1998; Davenport, 1998) nesse ambiente. Por outro lado, tendo em vista a ideia da inseparabilidade da comunicação científica das atividades a que serve, ressaltado por Goffman e Warren (1980) e Meadows (1999), a comunicação científica pode ser definida como um complexo sistema que viabiliza os fluxos da informação científica entre pesquisadores, de modo que estes possam, em uma dinâmica cíclica, acessar, usar, gerar e disseminar informação durante a realização de suas atividades como pesquisadores. A partir de dessa definição, considera-se que a comunicação científica e a gestão da informação científica estão inexorável e funcionalmente unidas. A primeira promove/gera os fluxos de informação enquanto que a segunda os sistematiza.

$\mathrm{Na}$ relação entre comunicação científica e gestão da informação científica destaca-se a perspectiva da interdependência e complementaridade. De um lado a gestão da informação científica que pressupõe, além do entendimento do ambiente em que os principais atores da comunidade científica estão inseridos (Birdsall, 2005; Mikhailov et al., 1984; Shearer e Birdsall, 2002), o envolvimento com processos e estruturas de comunicação científica. Estes, por sua vez, promovem, com a legitimidade conferida pela comunidade científica, o fluxo da informação na ciência. Do outro lado, o própria comunicação científica, que, per se, não dispõe de estratégias, mecanismos e procedimentos necessários para lidar com a sistematização requerida pelo volume crescente de informação científica, especialmente em ambiente digital, de modo que suas funções sejam efetivamente alcançadas. Nenhuma das abordagens é capaz de lidar, isoladamente, com questões estruturais emergentes que dizem respeito ao acesso e disseminação da informação científica. Essas questões surgem exatamente de deficiências ou limitações existentes tanto da gestão da informação científica quanto na comunicação científica, elencadas no Quadro 1. 
Limitações para promoção do acesso e disseminação da informação científica

Comunicação científica

Demandas relacionadas com o aumento da visibilidade e do impacto dos resultados de pesquisa, provenientes da comunidade científica, em função das quais torna-se imperativo o deslocamento da ênfase nos sub-processos organização, armazenamento e preservação da informação para os sub-processos de disseminação e promoção de seu uso. Tais demandas foram identificadas em obras de pesquisadores como, por exemplo, Borgman (2007), Houghton, Steele e Henty (2003), Swan (2004, 2006) Swan e Brown (2004, 2005).

Desenvolvimento de tecnologias, metodologias e mecanismos que correspondam às especificidades da informação científica, de seu fluxo e do seu contexto de geração e uso

Volume crescente da informação científica e emergência do digital como formato predominante para o acesso e disseminação da informação científica (Borgman, 2007).

Diversificação de suporte para a veiculação da informação científica (Houghton, Steele e Henty, 2003).

Gestão da informação científica

Restrições de acesso e disseminação de resultados de pesquisa publicados em artigos de periódicos científicos, impostas pelo modelo de direito de cópia, o qual preconiza que o autor ceda direitos patrimoniais exclusivos aos editores. Isso conduz ao monopólio do sistema por editores científicos comerciais que impõem custos exorbitantes às assinaturas de periódicos ao ponto que nem mesmo instituições de países ricos são capazes viabilizar a manutenção de suas coleções (Brody et al., 2004; Costa, 2006; Declaração de Berlin, 2003; Jacobs, 2006; Suber, 2007; Willinsky, 2006).

Mudanças nas atividades de produção do conhecimento científico, decorrentes, sobretudo, do uso crescente de tecnologias de informação e comunicação. Tais mudanças, além de interferir nas maneiras como a pesquisa científica é conduzida, requerem transformações nos modos como seus resultados são gerenciados e comunicados (Houghton, Steele e Henty, 2003).

Aumento crescente das atividades científicas, e, consequentemente, do volume de informação científica produzida e disseminada, principalmente em formato digital.

Necessidade de acesso amplo a uma variedade cada vez maior de recursos e fontes de informação de modo a subsidiar a produção do conhecimento científico e que, além disso, transcendam limites disciplinares e favoreçam a interação entre áreas do conhecimento (Houghton, Steele e Henty, 2003; Maron e Smith, 2008).

Demanda de uso de tecnologias de informação e comunicação como suporte ao trabalho colaborativo entre pesquisadores e instituições (Borgman, 2007; Haridasan e Khan, 2009; Hine, 2006; Olson et al., 2008).

Necessidade de armazenamento, preservação, acesso, disseminação e reutilização de recursos informacionais não convencionais que, do mesmo modo, resultam das atividades de pesquisas como, por exemplo, conjuntos de dados brutos de pesquisa, simulações, software, objetos multimídia e outros (Houghton, Steele e Henty, 2003).

Os aspectos em destaque figuram entre os principais que se impõem como fatores limitantes para que ambas as abordagens - gestão da informação científica e comunicação científica - a partir de processos, mecanismos e estratégias próprias, respondam satisfatoriamente às necessidades da comunidade científica. Ou seja, nem uma nem outra prática, isoladamente, dispõe de ferramental suficiente para lidar com o cenário atual dos fluxos de informação que alimentam e que resultam das atividades de pesquisa. Entretanto, em razão dos entraves do sistema de comunicação científica tradicional, como aqueles que fizeram culminar a crise dos periódicos, a própria comunidade científica empreendeu esforços em direção à remoção de barreiras ao flu- 
xo da informação científica, resultando no movimento mundial em favor do acesso aberto (Odlyzko, 2006; Willinsky, 2006). O acesso aberto é responsável pela reestruturação de processos de comunicação científica relacionados com a produção, disseminação e uso do conhecimento. A despeito de suas motivações primárias estarem ligadas a tais aspectos, sua operacionalização somente é viável pelo fato de que suas ações estarem pautadas por processos de gestão da informação científica, conforme denotam características e estratégias apontadas por diversos autores (Brody et al., 2004; Costa, 2006; Declaração de Berlin, 2003; Jacobs, 2006; Suber, 2007; Willinsky, 2006). Considerando a vinculação funcional entre acesso aberto e gestão da informação científica, assume-se que, para otimizar o fluxo da informação científica, reformulando processos de comunicação científica, o acesso aberto recorre a processos sistematizados de gestão da informação científica.

Desse modo, conforme representado na Figura 1, parte-se do pressuposto que, na abordagem do acesso aberto, a solução de problemas de comunicação científica passa, necessariamente, pelo gerenciamento apropriado da informação científica. Esta, por sua vez, deve considerar aspectos próprios da comunicação científica - como é o caso das estratégias de acesso aberto, como esforço de melhoria dos processos de comunicação da informação no contexto científico. Nesse sentido, como já destacado, revela-se a relação de interdependência e complementaridade entre as duas abordagens, cuja intersecção corresponde ao acesso aberto, conforme ilustrado na Figura 2. Portanto, a análise da literatura proveniente das duas abordagens permitiu sugerir que o acesso aberto constitui a intersecção existente entre a gestão da informação científica e a comunicação científica.

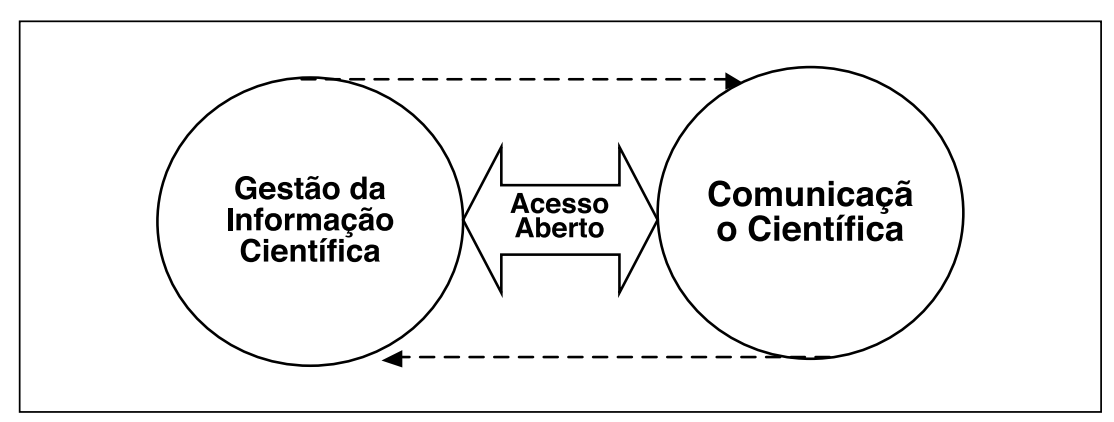

Figura 1. Relacionamento entre os tópicos gestão da informação científica e comunicação científica e acesso aberto

Fonte: Elaboração própria. 


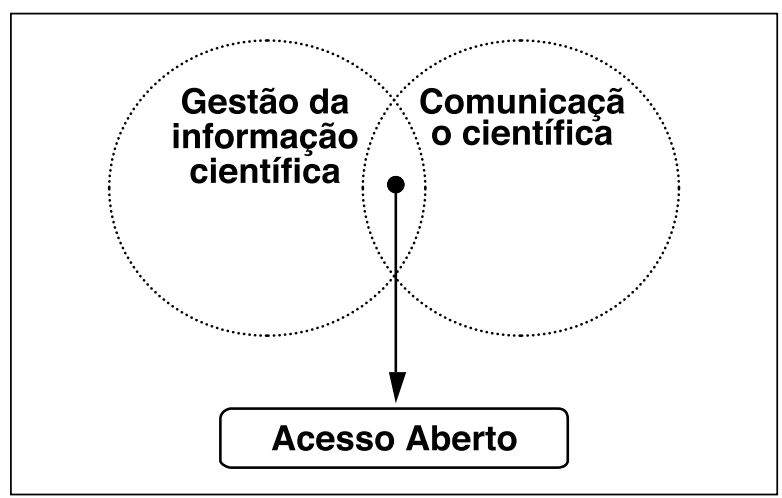

Figura 2. Acesso aberto como a intersecção entre gestão da informação científica e comunicação científica

Fonte: Elaboração própria.

\section{Metodologia}

Trata-se de uma pesquisa de abordagem mista, ou seja, foi operacionalizada com base na combinação de métodos qualitativos e quantitativos para a coleta e análise dos dados. Nesta perspectiva, adotou a estratégia de triangulação concomitante, onde dados quantitativos e qualitativos foram coletados simultaneamente e, em seguida, integração e comparados lado a lado. A pesquisa teve como sujeito pesquisadores vinculados aos institutos de pesquisa do Ministério da Ciência, Tecnologia e Inovação (MCTI). Estabeleceu-se como parâmetro para a escolha de duas instituições a aplicação dos critérios produtividade científica e representação de diferentes divisões do conhecimento. Para o primeiro critério, adotou-se a quantidade de recursos de informação indexados na plataforma Web of Knowledge. De acordo com a plataforma, entre os institutos de pesquisa vinculados ao MCTI, o Centro Brasileiro de Pesquisas Físicas (CBPF) encabeçou a lista, e, portanto, foi selecionado para o estudo dentro do critério produtividade. Da aplicação do primeiro critério para a definição da amostra, que resultou na escolha do CBPF, foi possível aplicar o segundo critério, que foi a representação de diferentes divisões do conhecimento. Por contemplar tanto disciplinas das ciências sociais quanto das humanidades, do ponto de vista das práticas de produção do conhecimento, o MAST foi considerado o instituto de pesquisa que mais se diferencia do CBPF. Desse modo, a amostra foi constituída de todos os pesquisadores doutores das ambas as instituições. O modelo genérico de gestão da informação científica fundamentado na perspectiva da comunicação científica e do 
acesso aberto, objetivo maior da pesquisa, foi construído com base nos dados coletados a partir de três estratégias distintas, resumidas na Figura 3.

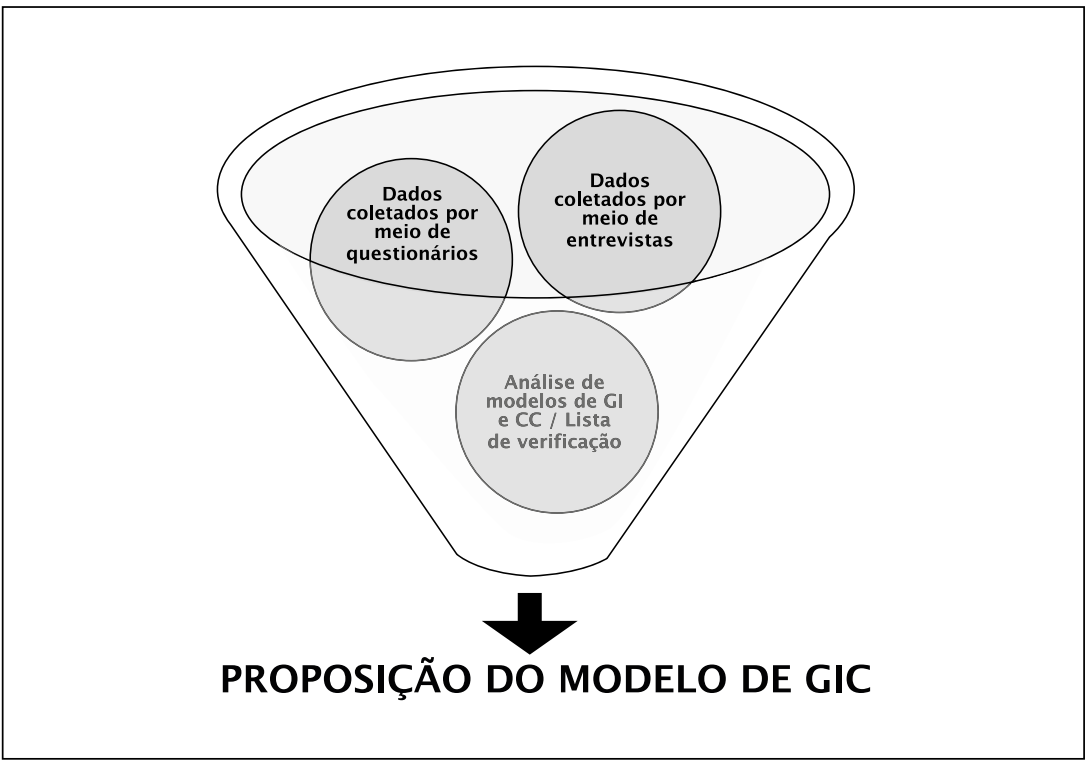

Figura 3. Procedimentos para a coleta de dados para a proposição do modelo de GIC Fonte: Elaboração própria.

Os resultados apresentados a seguir dizem respeito ao modelo proposto resultante da pesquisa realizada. Resultados da investigação a partir da qual o modelo foi derivado encontram em Leite (2011), onde é possível realizar uma leitura detalhada que permite verificar a origem de cada um dos seus elementos e das relações entre eles.

\section{MODELO DE GESTÃO DA INFORMAÇÃO CIENTÍFICA}

\section{Locus: os institutos de pesquisa}

O modelo proposto tem como locus os próprios institutos de pesquisa, vistos como um sistema aberto (espaço cor-de-rosa da representação gráfica). Os institutos de pesquisa interagem e intercambiam recursos com seu ambiente, dentre os quais está a informação científica, um dos principais insumos e resultados de suas principais atividades: a produção do conhecimento científico. 


\section{Fluxo da informação científica}

A entrada e a saída de informação científica dos institutos de pesquisa (cujos requisitos foram identificados a partir da análise de necessidades e atividades de busca, acesso e uso da informação e hábitos de comunicação) dependem das relações estabelecidas com o ambiente em que atuam. Dentre as formas de viabilizar tais relações estão os processos realizados pelo sistema de comunicação científica, que é responsável por todos os aspectos que dizem respeito ao fluxo da informação científica. Significa dizer que a informação que alimenta e que resulta das atividades de pesquisa (conceito derivado da análise e discussão dos resultados da pesquisa) tem seu fluxo promovido por processos de comunicação científica. Modelos que representam o sistema de comunicação científica (Birdsall, 2005; Shearer e Birdsall, 2002; Mikhailov et al., 1984), assim como modelos que representam processos de comunicação científica (Garvey e Griffith, 1979; Hills, 1983; Houghton et al., 2009; Hurd, 1996, 2000, 2004; Lancaster e Smith, 1978; Mikhailov et al., 1984; Søndergaard et al., 2003; UNISIST, 1971) ilustram o aspecto da entrada e da saída de informação científica no contexto de instituições de pesquisa. Além disso, tal dinâmica foi detectada de dados obtidos por meio de entrevistas e questionário (busca, acesso, uso e comunicação da informação) e lista de verificação (comunicação da informação). A informação e a comunicação científica estão representadas na versão gráfica do modelo pelas formas em alaranjado.

\section{Contexto determinante: $o$ acesso aberto}

Dados os principais obstáculos que tornam difícil o alcance das funções da comunicação científica, o acesso aberto constitui um imprescindível componente (forma de cor verde na versão gráfica). Trata-se do principal componente que contribui para a retomada e garantia dos fluxos desimpedidos de informação que alimentam, tal como enfatizado por pesquisadores sujeitos da investigação, e resultam das atividades de pesquisa, necessários ao desenvolvimento da ciência, tal como enfatizado por pesquisadores na seção "Processos de gestão da informação científica", e como aquilo que o modelo tradicional de comunicação científica já não proporciona (Brody et al., 2004; Costa, 2006; Declaração de Berlin, 2003; Jacobs, 2006; Suber, 2007; Willinsky, 2006). Na congregação de suas diferentes dimensões, representados pelos elementos de cor amarela, os esforços do acesso aberto são úteis e necessários à livre circulação da informação científica, em concordância com as motivações de pesquisadores para disseminar resultados de pesquisa, discutidos nos resultados da pesquisa, um dos principais elementos propulsores das atividades de produção do conhecimento. 


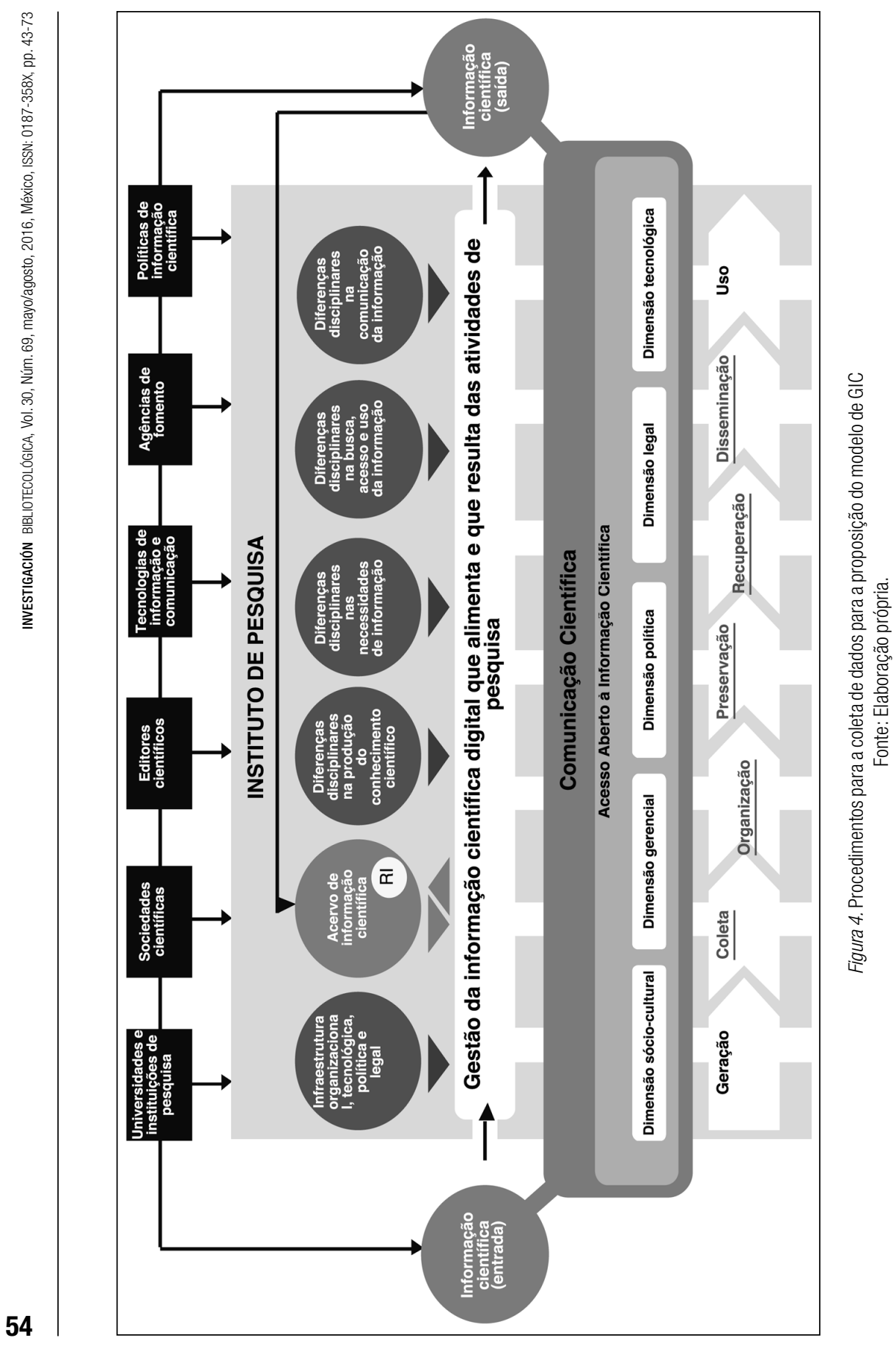


$\mathrm{Na}$ perspectiva do modelo proposto quanto a promoção de fluxos desimpedidos de informação científica, o acesso aberto age sobre duas principais situações fundamentais. A primeira situação diz respeito à criação de condições para que pesquisadores possam buscar, acessar e usar toda a informação que necessitam para desenvolver seu trabalho, atividades exploradas da investigação. A segunda situação refere-se à garantia de condições para que os resultados das pesquisas realizadas circulem e sejam rapidamente utilizados por outros pesquisadores, dentro e fora da instituição, favorecendo a geração de novos conhecimentos. Aspectos relacionados com essa atividade foram explorados no estudo sobre hábitos de comunicação. Nessas duas situações fundamentais a contribuição do acesso está sobre a aceleração da produção de conhecimento, aumento do impacto dos resultados de pesquisa e de sua visibilidade e de seus geradores (Brody et al., 2004; Costa, 2006; Declaração de Berlin, 2003; Jacobs, 2006; Suber, 2007; Willinsky, 2006).

\section{Dimensóes do acesso aberto}

Os institutos de pesquisa necessitam lidar com a informação científica que é necessária e que resulta das atividades de pesquisa. É imperativo satisfazer as necessidades de informação dos pesquisadores, informação esta que se encontra dentro e fora dos institutos. Ao mesmo tempo, é preciso promover amplamente a circulação da informação que resulta de suas atividades. Para tanto, o modelo de gestão da informação científica proposto leva em consideração a comunicação científica fundamentada nas estratégias de acesso aberto, que agem nas duas situações, segundo resultados da pesquisa. É oportuno ressaltar que, no modelo proposto, os esforços de acesso aberto são conduzidos tendo em vista suas diferentes dimensões, que, somadas, resultam em ações robustas. Cada uma dessas dimensões é sucintamente descrita a seguir.

- Dimensão sócio-cultural: o acesso aberto requer mudança de comportamento entre os atores envolvidos. O comportamento de pesquisadores frente ao sistema de comunicação científica foi moldado e legitimado a partir normas de convivência estabelecidas ao longo dos tempos. Além disso, variam também em razão das próprias diferenças existentes entre as disciplinas (Antelman, 2004, 2006). Com isso, a transformação de atividades, o compartilhamento de funções que antes era executadas por determinados atores, ou mesmo a inserção de um determinado processo antes inexistente no sistema de comunicação científica requer a observação dos comportamentos vigentes. Isso é 
importante, inclusive, para o estabelecimento das ações intervenientes necessárias no contexto social e cultural em que estão inseridos. Como exemplo, cabe mencionar o autoarquivamento da produção científica, que requer interferências políticas e legais no contexto sociocultural em que se inserem pesquisadores (Crow, 2002);

- Dimensão gerencial: a implementação do acesso aberto no bojo de um modelo de gestão da informação requer atividades de planejamento, organização, direção e controle, de modo que os objetivos sejam efetivamente alcançados. Nessa perspectiva, a implementação da via dourada e da via verde para o acesso aberto, também como estratégia constituintes de esforços de gestão da informação científica, requer a coordenação de processos gerenciais que contribuam para que seus benefícios sejam alcançados. A ideia da aplicação de funções administrativas em processos de gestão da informação é recorrentemente mencionada na literatura (Diener, 1992; Fairer-Wessels, 1997; Vickers, 1985; Wilson, 2002) e se justifica no modelo uma vez que o acesso aberto envolve recursos, processos, sistemas e pessoas que necessitam ser geridos apropriadamente;

- Dimensão política: o acesso aberto depende de normas que suportem institucionalmente suas ações. Dentre as normas mais relevantes estão aquelas que tornam obrigatório o arquivamento da produção científica em ambientes de acesso aberto. Estas são estabelecidas por universidades, institutos de pesquisa e agências de fomento. No contexto dos repositórios institucionais de acesso aberto, são os mandatos de depósito os responsáveis pelas altas taxas de povoamento (Carr et al., 2006; Harnad, 2006). Ou seja, aquelas universidades ou institutos de pesquisa que o estabeleceram, alcançam praticamente $100 \%$ da produção científica depositada. Além dos instrumentos normativos, são fundamentais também as estratégias de apoio político às iniciativas de acesso aberto, seja em direção ao convencimento de comunidades, seja aprimorando e qualificando processos gerenciais.

- Dimensão legal: diz respeito, sobretudo, ao estímulo ou determinações para que pesquisadores publiquem os resultados de suas pesquisas em veículos de acesso aberto (via dourada) ou que permitam o arquivamento em repositórios (via verde). Mais do que isso, tais mecanismos legais preveem que os autores retenham seus direitos de cópia e que estes sejam cedidos não exclusivamente à própria instituição (Bailey, 2006; Moore, 2011; Suber, 2010). Desse modo, é possível disseminar amplamente a produção científica sem constrangimentos de qualquer natureza. Em sentido amplo, esta dimensão foi objeto de discussão 
a partir de dados coletados tanto por meio de entrevistas quanto de questionário. Constatou-se que, embora seja um aspecto apontado como central no acesso aberto, pesquisadores não a importância da retenção de direitos de cópia dos trabalhos por parte dos autores.

- Dimensão tecnológica: de um modo geral esta dimensão requer que as iniciativas de acesso aberto acompanhem e desenvolvam-se sob a luz da iniciativa de arquivos abertos, primando pelos padrões de interoperabilidade entre sistemas (Costa, 2006; Crow, 2002; Hurd, 2004; Suber, 2010) Além disso, a adoção de software livre tem sido ocorrido amplamente em todo o mundo.

\section{Processos de gestão da informação científica}

Diante dos inúmeros desafios em lidar com o ambiente informacional do qual fazem parte os institutos de pesquisa, há a necessidade de sistematizar e controlar os fluxos de informação científica em nível institucional, tanto daquela que alimenta quanto daquela que resulta das atividades de pesquisa, de modo a maximizar seus benefícios interna e externamente. Para tanto, faz-se necessária a institucionalização de processos de gestão da informação científica.

Tal como em outros contextos, os processos de gestão da informação científica correspondem a um ciclo. Ou seja, um conjunto de fases interconectadas e interdependentes que se repetem sucessivamente em uma ordem estabelecida. O modelo proposto assume a perspectiva processual tanto de modelos de gestão da informação registrados na literatura (Choo, 1998, Davenport, 1998; Detlor, 2009) quanto de modelos de processos de comunicação científica (Garvey e Griffith, 1979; Hills, 1983; Houghton et al., 2009; Hurd, 1996, 2000, 2004; Lancaster e Smith, 1978; Mikhailov et al., 1984; Søndergaard et al., 2003; UNISIST, 1971). Dessa forma, compreende os processos específicos de geração, coleta, organização, preservação, recuperação, disseminação e uso da informação necessária e criada a partir das atividades de pesquisa dos institutos de pesquisa. Com base nos elementos e definições presentes nos modelos mencionados, tais processos estão sucintamente definidos a seguir a partir de resultados obtidos nesta pesquisa:

- Geração: diz respeito aos processos de geração da informação científica, que tem início em resultados das atividades de pesquisa que são consolidadas a partir de processos editoriais de modo a resultar na literatura científica. Características dos processos de geração do conhecimento, assim como a própria produção da informação com vistas à 
comunicação foram discutidas ao longo dos resultados da pesquisa realizada;

- Coleta: corresponde à aquisição dos recursos de informação científica que é necessária para fazer pesquisa e que resulta delas. Trata-se dos esforços empreendidos em capturar informação científica a ser gerenciada pelo sistema. Características desse processo foram exploradas ao longo dos resultados da pesquisa realizada;

- Organização: diz respeito aos processos e utilização de instrumentos de representação dos recursos de informação científica com vistas à sua posterior recuperação pelos usuários. Aspectos relacionados com esse processo surgiram em citações dos entrevistados;

- Preservação: conjunto de atividades de cunho tecnológico e gerencial que contribuem para garantir o acesso permanente e a longo prazo à informação em suporte digital;

- Recuperação: processo realizado a partir de uma interface de busca onde os usuários elaboram de estratégias de busca, cuja execução, por meio do sistema de recuperação, resulta inicialmente na apresentação dos registros que representam recursos de informação, e, em seguida, ao seu inteiro teor, correspondentes às suas necessidades de informação. Aspectos relacionados com esse processo surgiram em citações dos entrevistados;

- Disseminação: corresponde aos esforços e mecanismos para fazer fluir amplamente recursos de informação científica e facilitar sua descoberta e uso, contribuindo para a visibilidade dos resultados de pesquisa, do pesquisador e da instituição. Aspectos relacionados com esse processo surgiram em citações dos entrevistados;

- Uso: processo que precede e está intimamente relacionado com a geração da informação. Diz respeito ao consumo da informação manifesta na literatura científica.

\section{Influências do acesso aberto e de forças externas sobre os processos de gestão da informação científica}

Os processos específicos de gestão da informação científica, sob a égide da perspectiva sistêmica da comunicação científica e do acesso aberto, recebem influência direta do ambiente interno e externo dos institutos de pesquisa. Isso quer dizer que:

- Geração: esse processo é influenciado em diferentes aspectos. Primeiro, na medida que a pesquisa é realizada colaborativamente, como per- 
cebido no estudo, argumenta-se que há uma influência externa direta, seja de pesquisadores, grupos ou mesmo instituições (Fry, 2006; Houghton, Steele y Henty, 2003; Hurd, 1996; Jamali e Nicholas, 2008; Katz e Martin, 1997; Swan, 2008). Os resultados da pesquisa refletem esta situação que se manifesta em tipo de autoria e, sobretudo, em decisões de quando, onde (o que determina tipos os detentores dos direitos de cópia e licenças), o que publicar e como fazer disseminá-los (dependendo de onde foi publicado). Além disso, a maior ou menor exposição de usuários à sistemas de informação de acesso aberto influenciam a geração de resultados de pesquisa;

- Coleta: a coleta de informação científica ocorre tanto nos acervos dos próprios institutos de pesquisa, quanto no ambiente externo a eles, como pôde ser observado em alguns modelos de comunicação científica (Houghton et al., 2009; Søndergaard et al., 2003; UNISIST, 1971) e em funções desempenhadas por atores presentes no sistema de comunicação científica (Birdsall, 2005; Mikhailov et al., 1984; Shearer e Birdsall, 2002). A noção do processo de coleta de informação, e as influências externas sobre ele, além de encontrarem fundamento na literatura, são sustentados também em aspectos revelados pela investigação das atividades de busca, acesso e uso (análise e discussão dos dados da pesquisa). A construção de um mecanismo de busca temático (provedor de serviços na principal área de atuação e de áreas correlatas do instituto de pesquisa) baseado na perspectiva do acesso aberto permite a sistematização e a coleta automatizada e monitoramento de recursos de informação disponíveis e acessíveis online na Internet. Esses mecanismos de busca monitoram tanto periódicos científicos de acesso aberto, quanto repositórios institucionais, repositórios disciplinares, bibliotecas digitais e portais de conferências de acesso aberto, dentre outros possíveis recursos. Além disso, é necessário estimular o uso de mecanismos de buscas multidisciplinares (provedores de serviços), que realizam buscas cruzadas, com a intenção de suportar demandas de informação de atividades de pesquisa de natureza interdisciplinar;

- Organização: trata-se de um processo fortemente influenciado pela perspectiva do acesso aberto, uma vez que se torna imprescindível a adoção de padrões internacionais de interoperabilidade entre sistemas (Borgman, 2007; Hurd, 2004; Lagoze e Van de Sompel, 2001). Tais padrões referem-se principalmente a esquemas de metadados e instrumentos auxiliares de representação da informação, protocolos de comunicação. No contexto do acesso aberto à informação científica são adotados vários esquemas padronizados de metadados, como 
por exemplo o Dublin Core, e o Protocolo para a Coleta de Metadados da Iniciativa de Arquivos Abertos (OAI-PMH). Nesse caso, muito embora o OAI-PMH não se refira a qualquer processo de representação da informação, sua presença no processo de organização é justificável. Infraestruturas desse tipo são capazes de permitir a exposição de metadados das coleções de recursos de informação científica de modo a permitir sua coleta por outras instituições;

- Preservação: embora o acesso aberto à informação científica não tenha como principal objetivo a preservação da informação digital, mas sim a maximização dos impactos de pesquisa por meio da maximização do seu acesso a uso, algumas de suas estratégias necessariamente a promovem. Repositórios institucionais de acesso aberto à informação científica, caso sejam bem planejados, além de terem como uma de suas linhas de atuação a preservação da produção científica da instituição, criam condições férteis e objetivas para o desenvolvimento de programas de preservação digital baseadas em modelos internacionalmente recomendados (Crow, 2002; Lynch, 2003). Do mesmo modo, a adoção de identificadores persistentes contribuem para a preservação do acesso permanente aos recursos de informação;

- Recuperação: a recuperação da informação é potencializada caso o sistema tenha por base mecanismos de acesso aberto e interoperáveis (Borgman, 2007; Crow, 2002; Hurd, 2004; Lagoze e Van de Sompel, 2001; Lynch, 2003). Tais mecanismos, além de possuírem relação direta com o processo de organização da informação, promovem melhores condições para que recursos de informação tornem-se mais encontráveis e recuperáveis por diversos sistemas distribuídos em todo o mundo. Nesse sentido, a partir de uma única interface, ou mesmo poucas interfaces, usuários podem realizar buscas simultâneas em centenas de provedores de dados (instituições que alimentam e mantém os ambientes de acesso aberto) mesmo desconhecendo autores ou as instituições que os mantém. A recuperação da informação na perspectiva do acesso aberto beneficia ao mesmo tempo a descoberta facilitada de informação para pesquisadores dos institutos, ao tempo que torna sua produção científica encontrável e recuperável em todo o mundo. Este ponto tem a ver com o próximo processo;

- disseminação: na perspectiva do acesso aberto, sistemas de informação passam a atender a demandas dos pesquisadores por aumento da visibilidade de sua produção científica e de si mesmos (Brody et al., 2004; Costa, 2006; Declaração de Berlin, 2003; Jacobs, 2006; Suber, 2007; Willinsky, 2006). Este aspecto talvez tenha sido um daqueles 
que bibliotecas e centros de documentação mais mantinham distância durante muito tempo, pois se tratava de uma prerrogativa, sobretudo, de editores de publicações científicas. A partir do desenvolvimento de ações do acesso aberto, muitas dessas funções foram alteradas (Crow, 2002). As instituições dos pesquisadores, por meio de seus serviços de informação, passaram a atuar sobre a construção de gerenciamento de vias alternativas de comunicação científica, como aquelas representadas por repositórios institucionais. Esses serviços de informação passaram então a não apenas constituir e organizar acervos de informação, mas principalmente, como nunca antes, disseminá-los amplamente, graças ao modo como os processos de gestão da informação científica anteriores à disseminação foram estruturados. Nessa forma de atuação é possível atender às demandas por promoção da visibilidade dos resultados de pesquisa, do pesquisador e da própria instituição. Aspectos relacionados com esse processos foram analisados e discutidos na pesquisa realizada, mais especificamente na análise e discussão dos dados sobre hábitos de comunicação;

- Uso: todos os processos anteriores visam, em última análise, promover o uso da informação científica de modo que novos processos de geração de conhecimento sejam iniciados. Este constitui um dos objetivos imediatos do acesso aberto, que é aumentar o impacto dos resultados de pesquisa, leia-se, aumento das taxas de citação, por meio da maximização do seu acesso e uso (Harnad e Brody, 2004; Swan, 2010). Ou seja, na medida que os processos anteriores são potencializados pelo o acesso aberto, o uso também o é.

Nesse momento é importante frisar que os processos de coleta, organização, preservação, recuperação e disseminação são conduzidos a partir da implementação de repositório institucional de acesso aberto à informação científica (Crow, 2002, Costa, 2006; Suber, 2007). Por esta razão, na versão gráfica do modelo de gestão da informação científica, tais processos estão destacados e em verde. Esta é a mesma cor que representa o repositório institucional sinalizado, um círculo verde inserido no elemento acervo de informação científica institucional. Desse modo, a informação científica que resulta das atividades de pesquisa no instituto de pesquisa é incorporada ao acervo de informação institucional, a partir de seu repositório. E é a partir desse mesmo repositório institucional que os processos de gestão da informação científica em destaque (verde) são realizados. 


\section{Elementos flexíveis do modelo}

Por outro lado, além da influência direta do da comunicação científica e do acesso aberto, o modelo de gestão da informação científica também prevê a influência de um conjunto de elementos internos ao ambiente dos institutos de pesquisa. Esses elementos constituem as partes flexíveis do modelo, ou seja, aquelas que variam em razão dos ambientes institucionais e das diferenças disciplinares existentes entre as áreas do conhecimento, conforme indicação dos resultados presentes na seção de análise e discussão. É importante ressaltar que o modelo é genérico. Por esta razão, prevê a influência de tais elementos, porém, não considera em sua constituição a explicitação de particularidades da infraestrutura da instituição nem tão pouco as diferenças disciplinares relacionadas com necessidades, busca, acesso uso e hábitos de comunicação da informação.

Até então, todos os elementos descritos, assim como as relações entre eles, constituem partes presentes do modelo genérico, que tem nas partes flexíveis mais alguns de seus componentes. Tais partes correspondem às representações circulares internas ao instituto na versão gráfica do modelo. Cada um desses elementos e o modo como influenciam a gestão da informação científica são explorados a seguir:

- Infraestrutura organizacional, tecnológica, política e legal: essa força de influência aglutina um conjunto de aspectos fundamentais para a gestão da informação científica. No quesito infraestrutura organizacional, presume-se a existência de departamento ou área específica cujas funções primordiais estejam relacionadas com atividades informacionais (Fairer-Wessels, 1997; Detlor, 2009; Søndergaard et al., 2003; UNISIST, 1971). Normalmente essas funções estão reunidas em torno de bibliotecas de pesquisa ou centros de documentação (Birdsall, 2005; Lancaster e Smith, 1978; Shearer e Birdsall, 2002). Decorrente disso, presume-se que a infraestrutura organizacional requer suporte financeiro e recursos humanos qualificados não apenas em relação às técnicas de gestão da informação mas também quanto ao funcionamento das comunidades científicas, da comunicação científica e do acesso aberto. No quesito infraestrutura tecnológica, estão inseridos todos os aspectos inerentes à provisão da malha tecnológica e de redes necessária à implantação do modelo de gestão da informação científica (Choo, 1998; Detlor, 2009; Fairer-Wessels, 1997; Rowley, 1998). Isso requer o conhecimento técnico de software livres e padrões utilizados em iniciativas de acesso aberto bem como também é necessária a ciência 
dos analistas quanto ao funcionamento das comunidades científicas, da comunicação científica e do acesso aberto (Borgman, 2007; Brody et al., 2004; Costa, 2006; Jacobs, 2006; Hurd, 2004; Lagoze e Van de Sompel, 2001; Willinsky, 2006; Suber, 2007). A infraestrutura política, por seu turno, encerra uma importante função na governança da gestão da informação científica. A legitimação corporativa do alcance dos novos processos de gestão da informação científica, ou mesmo da mudança de processos já existentes, depende de sua institucionalização formal, a ser promovida por instância de nível estratégico. Exemplo disso são as políticas institucionais de acesso aberto, que podem, ao mesmo tempo, estimular a publicação de resultados de pesquisa em veículos de acesso aberto e requerer que a produção científica de seus pesquisadores seja depositada em repositório institucional de acesso aberto (Bailey, 2006; Carr et al., 2006; Moore, 2011; Harnad, 2006; Suber, 2010). Ações políticas, incluindo as de convencimento, provenientes também dessas instâncias junto aos pesquisadores, são fundamentais para que a gestão da informação científica, tal como prevista pelo modelo, possa ser instituída e praticada na organização. A infraestrutura legal, por seu turno, constitui uma importante condição que viabiliza ou inviabiliza a prática de gestão da informação científica. Uma situação comum em instituições de pesquisa é a coexistência da pressão por produtividade científica (publicação) e os conflitos decorrentes da cessão de direitos patrimoniais dos resultados de pesquisa publicados, sobretudo, em artigos de periódicos científicos (Bailey, 2006; Moore, 2011; Suber, 2010). Ou seja, as instituições passam a não ter controle sobre aquilo que produziram em razão da pressão, exercida por ela mesma, por publicação em periódicos internacionais de prestígio que, via de regra, tomam de assalto os direitos de cópia em troca da publicação. Isso é um círculo vicioso que tende a ser interrompido à medida que a gestão da informação científica orientada pelo acesso aberto passa a vigorar. Quer-se dizer com isso que os institutos de pesquisa devem conscientizar, estimular e instrumentalizar seus pesquisadores a negociarem os direitos de cópia no momento publicação de seus trabalhos, quando, evidentemente, estes puderem estar comprometidos. Ou mesmo estimular a publicação em veículos de acesso aberto, que já preveem tal condição. Além e mais do que isso, os institutos de pesquisa devem estabelecer normas que prevejam que seus pesquisadores cedam direitos não exclusivos de distribuição de seus trabalhos em formato digital na Internet. Para as atividades editoriais sob responsabilidade do próprio instituto de pesquisa, como é 
o caso da publicação de periódicos científicos, livros, séries e outros produtos de informação, é mister que sejam incorporadas licenças e permissões que favoreçam à sua livre circulação, como que o caso de algumas combinações de licenças Creative Commons ou similares (Suber, 2010). Aspectos desse elemento flexível têm origem nas sentenças derivadas da análise e discussão dos resultados da pesquisa;

- Acervo de informação científica institucional: os acervos de informação científica das instituições devem ser formados em razão de dois critérios fundamentais elementares recorrentes no modelo proposto: a informação que é necessária para fazer pesquisa e a informação que resulta das atividades de pesquisa (Birdsall, 2005; Lancaster e Smith, 1978; Roosendaal e Geurts, 1997; Shearer e Birdsall, 2002). Historicamente, as bibliotecas e centros de documentação vinham atuando principalmente no primeiro momento, ou seja, reunindo e permitindo acesso, mal ou bem, aos recursos de informação necessários à realização da pesquisa (Søndergaard et al., 2003; UNISIST, 1971). Por outro lado, uma vez que maior parte dos resultados de pesquisa são publicados fora da instituição, sob a lógica do sistema tradicional de publicação científica, as bibliotecas ou centros de documentação enfrentavam dificuldade para reunir e permitir acesso à produção científica de autoria de pesquisadores da instituição. A emergência do acesso aberto, como novo paradigma da comunicação e certamente também da gestão da informação científica, transformou determinadas funções e deu à esses serviços de informação condições para gerenciar a informação científica de autoria de pesquisadores da instituição por meio dos repositórios institucionais de acesso aberto (Borgman, 2007; Brody et al., 2004; Costa, 2006; Jacobs, 2006; Hurd, 2004; Lagoze e Van de Sompel, 2001; Willinsky, 2006; Suber, 2007). Ou seja, o acervo de informação científica de uma determinada instituição passa a contar também com processos de gestão da informação científica que resulta de suas atividades. A formação das coleções do repositório institucional são fundamentais que os resultados de pesquisa, pesquisadores e a própria instituição tenham sua visibilidade aumentada. Aspectos desse elemento flexível têm origem nas sentenças derivadas da análise e discussão dos resultados da pesquisa;

- Diferenças disciplinares na produção do conhecimento científico: o modo como pesquisadores conduzem suas atividades de pesquisa (Jamali e Nicholas, 2008; Houghton, Steele y Henty, 2003; Katz e Martin, 1997; Swan, 2008) influencia os processos de gestão da informação científica que resulta de tais atividades. A satisfação de necessidades de infor- 
mação de pesquisadores cujas atividades de investigação são mais ou menos interdisciplinares requer particularidades dos processos de coleta, recuperação e disseminação da informação científica (Houghton, Steele y Henty, 2003). Ou seja, áreas correlatas presentes em determinadas atividades demandam o suporte informacional também nessa perspectiva, o que exige sistemas de informação do mesmo modo mais ou menos interdisciplinares. $\mathrm{O}$ trabalho colaborativo, por envolver pesquisadores de outras instituições, também influencia determinados processos de gestão da informação científica. Isso ocorre porque impactam a geração da informação, o que, por sua vez, determina tipo de autoria, decisões de quando, onde, o que publicar e como disseminar. Aspectos desse elemento flexível têm origem nas sentenças derivadas da análise e discussão dos resultados da pesquisa;

- Diferenças disciplinares nas necessidades de informação: as necessidades de informação são influenciadas pelos contextos de atuação dos pesquisadores (Choo, 1998; Davenport, 1998; Rowley, 1998; Shearer e Birdsall, 2002). Como exemplo disso está a constatação, alcançada por meio dos levantamentos, de que físicos possuem necessidades de informação diferentes de cientistas sociais e humanistas. Evidentemente, nenhum modelo de gestão da informação científica poderá ser genérico caso tente explicitar em sua própria constituição as necessidades específicas de informação. Contudo, também como foi possível constatar, as atividades de pesquisa de qualquer área do conhecimento, invariavelmente, dependem de informação científica e culminam na geração de informação científica. Estes contornos gerais devem ser levados em consideração e previstos na proposta e estruturação de um modelo genérico de gestão da informação científica. Além disso, qualquer modelo deve prever que há essas especificidades que variam em função das diferenças disciplinares. Aspectos desse elemento flexível têm origem nas sentenças derivadas da análise e discussão dos resultados da pesquisa;

- Diferenças disciplinares na busca, acesso e uso da informação: os comportamentos associados à busca, acesso e uso da informação, são influenciados pelos contextos de atuação dos pesquisadores (Choo, 1998; Garvey e Griffith, 1979; Gorraiz et al., 2009; Houghton, Steele y Henty, 2003; Huang e Chang, 2008; Hurd, 2000). Do mesmo modo, como constatado na pesquisa, físicos possuem comportamento de busca, acesso e uso da informação diferente de cientistas sociais e humanistas. Essas diferenças influenciam o desenho de sistemas de informação científica para as áreas. Contudo, nenhum modelo de gestão 
da informação poderá ser genérico caso especifique em sua estrutura tais diferenças. Como se tratam de diferenças de natureza contextual, esse elemento está previsto no modelo proposto tendo em vista seu potencial de influência. Ou seja, padrões de busca, acesso e uso da informação devem ser previstos na implementação de um modelo dessa natureza. Aspectos desse elemento flexível têm origem nas sentenças derivadas da análise e discussão dos resultados da pesquisa;

- Diferenças disciplinares na comunicação da informação: os hábitos de comunicação da informação científica também variam em razão das áreas do conhecimento (Hurd, 2000; Houghton, Steele y Henty, 2003; Gorraiz et al., 2009; Huang e Chang, 2008). Pelas mesmas razões exploradas anteriormente, os hábitos de comunicação, que são empreendidas por pesquisadores de todas as áreas, invariavelmente, constituem um elemento influenciador da gestão da informação científica objeto do modelo proposto. Aspectos desse elemento flexível têm origem nas sentenças derivadas da análise e discussão dos resultados da pesquisa.

\section{Forças externas: elementos da comunidade científica}

Há outro conjunto de elementos que influencia as atividades dos institutos de pesquisa como um todo e também suas atividades de gestão da informação científica. São forças externas à instituição que representam atores ou mesmo tendências que impactam as atividades previstas no modelo de gestão da informação científica proposto, principalmente por estar fundamentado na comunicação científica e no acesso aberto. São seis grandes forças externas, identificadas em diversos modelos (Birdsall, 2005; Mikhailov et al., 1984; Shearer e Birdsall, 2002) e relacionadas com aspectos do acesso aberto (Borgman, 2007; Brody et al., 2004; Costa, 2006; Jacobs, 2006; Hurd, 2004; Lagoze e Van de Sompel, 2001; Willinsky, 2006; Suber, 2007), descritas a seguir:

- Universidades e instituições de pesquisa: são organizações que produzem e, por esta razão, consomem conhecimento e informação. Desse modo, constituem, ao mesmo tempo, fornecedoras e usuária da informação os institutos de pesquisa necessitam e geram, respectivamente. Comumente assumem papeis de colaboradoras em atividades de geração do conhecimento, o que, por sua vez, implica em comprometimentos no modelo de gestão da informação científica. Seus modelos de gestão da informação científica devem servir de benchmarking para a os esforços de gestão da informação científica do instituto de pesquisa; 
- Sociedades científicas: são entendidas como o agrupamento formal e representativo de pesquisadores que compartilham tópicos de estudo, desenvolvem pesquisas e se reúnem periodicamente. Constitui um dos ambientes em que pesquisadores compartilham resultados de suas pesquisas. Sua influência sobre o modelo de gestão da informação científica reside principalmente no fato de que tais sociedades além de representarem pesquisadores, catalisando suas aspirações, são muitas vezes responsáveis por publicações científicas, de acesso aberto ou restrito;

- Editores científicos: certamente uma das forças externas que mais exerce influência sobre o modelo de gestão da informação científica. Editores científicos influenciam diretamente tanto o fluxo da informação que alimenta as atividades de pesquisa quanto o fluxo da informação que resulta de tais atividades. Pesquisadores e suas instituições são produtores e usuários de informação científica. A informação científica, por sua vez, depende de editores científicos para poder se manifestar como literatura científica. A sua incorporação aos fluxos que alimentam e que resultam das atividades de pesquisa, seja na perspectiva do acesso aberto ou não, depende diretamente dos editores científicos, que são responsáveis pela consolidação dos resultados de pesquisa em informação científica;

- Tecnologias de informação e comunicação: os desenvolvimentos em tecnologias de informação e comunicação influenciam o próprio ciclo da informação, potencializando suas funções desde a geração até a utilização da informação. Esses avanços proporcionam novas possibilidades e oportunidades para processos informacionais. Exemplos claros disso são a aplicação da Internet nos processos de comunicação científica e, mais recentemente, toda a infraestrutura tecnológica que viabilizou o próprio acesso aberto à informação científica. Nesse contexto, desenvolvimentos em redes de banda larga, dispositivos móveis para acesso à informação, computação em nuvens, aperfeiçoamento de experiências entre humanos e computadores e de sistemas de recuperação de informação são eventos promissores.

- Agências de fomento: em uma perspectiva ampla, é possível que as agências de fomento sejam os atores que mais poder tenham em contribuir para o funcionamento efetivo de um sistema de gestão da informação científica fundamentado na comunicação científica e no acesso aberto. São muitos os exemplos de agências de fomento em todo o mundo que estabelecem suas políticas de acesso aberto. Essas políticas requerem que autores que recebem financiamento para suas pesquisas 
se comprometam em depositar seus resultados publicados ou aceitos para publicação em repositórios institucionais de acesso aberto ou que publiquem em periódicos de acesso aberto. Por esta razão, as políticas de acesso aberto instituídas por agências de fomento contribuem para a operacionalização tanto do acesso aberto por meio da via dourada quanto por meio da via verde;

- Políticas de informação cientifica: a articulação entre atores e variáveis presentes no contexto da produção e do uso da informação científica é objeto das políticas nacionais de informação em ciência e tecnologia. Normalmente, os atores constituem os mesmos presentes no sistema de comunicação científica. As variáveis, por outro lado, são aquelas relacionadas com a legislação e regulamentação, interesses dos diferentes atores e os contextos político- governamental, econômico e educacional. As políticas de informação científica são as responsáveis por provocar a sinergia entre os diferentes atores e variáveis. Por esta razão o estabelecimento de políticas de informação científica influenciam o funcionamento de um sistema de gestão da informação científica em qualquer contexto. Um exemplo de política de informação científica foi aquela em que a Coordenação de Aperfeiçoamento de Pessoal de Nível Superior (CAPES) em encerrar recursos para que bibliotecas mantivessem suas assinaturas de periódicos científicos e passou a promover o acesso eletrônico a um acervo de periódicos no modelo consórcio.

\section{Conclusões}

A ciência depende de fluxos de informação livres e desimpedidos para que possa se desenvolver efetivamente. O sistema de comunicação científica é responsável pelo fluxo da informação que alimenta e que resulta das atividades de pesquisa. Por esta razão, a comunicação rápida, adequada e eficiente dos resultados de pesquisas, transformados em informação, influencia diretamente o desempenho dos institutos de pesquisa, um dos atores que tem como principal função a geração de novos conhecimento científicos. Quanto mais rápida e completamente pesquisadores receberem a informação científica necessária às suas atividades, mais produtos científicos ele gerará a custos menores. Entretanto, como discutido ao longo deste trabalho, as funções da comunicação científica já não são alcançadas em razão de inúmeros desafios que são colocados no cenário informacional em que se inserem os institutos de pesquisa. 
Tendo em vista tais desafios, o modelo de gestão da informação científica tendo por base a comunicação científica e o acesso aberto foi proposto, visando a contribuir para que institutos de pesquisa possam responder de modo efetivo demandas emergentes de acesso, circulação e uso da informação científica. A solução, como indicado, perpassa pela intersecção de aspectos da gestão da informação, da comunicação científica e do acesso aberto à informação científica, atuando de forma integrada. Nenhuma dessas perspectivas isoladamente dispõe de instrumental teórico e metodológico para tratar dos problemas informacionais discutidos. Portanto, considera-se que o modelo proposto, além de um avanço no reconhecimento de como tais fenômenos podem ser observados e explicados, constitui um conjunto robusto de diretrizes norteadoras para a implementação da gestão da informação científica integrada à comunicação científica e ao acesso aberto.

É importante frisar que a construção do modelo proposto contou com diferentes estratégias para geração de dados que o sustentam (análise da literatura, análise e discussão de dados quantitativos e qualitativos) cujos resultados são apresentados por Leite (2011). É importante também mencionar a perspectiva genérica do modelo proposto, uma vez que seu delineamento considerou aqueles elementos gerais e necessários a qualquer modelo de gestão da informação científica e previu em sua constituição alguns elementos flexíveis, que acomodam possíveis diferenças contextuais que variam de instituto para instituto.

\section{REFERÊNCIAS}

Antelman, K. 2004. "Do open access articles have a greater research impact?". College and Research Libraries 65 (5), set.

_ 2006. "Self-archiving practice and the influence of publisher policies in the social sciences". Learned Publishing 19: 85-95.

Bailey, C. W. 2006. "What is open access?”, N. Jacobs, ed., Open access: key strategic, technical and economic aspects. Oxford: Chandos Publishing, 13-26.

Birdsall, W. F. 2005. Towards an integrated knowledge ecosystem: a canadian research strategy. Report submitted to the Canadian Association of Research Libraries/L'Association des bibliothèques de recherche du Canada (CARL/ABRC).

Borgman, C. L. 2007. Scholarship in the digital age: information, infrastructure, and the internet. Cambridge, Londres: MIT Press.

Brody, T. et al. 2004. "The effect of open access on citation impact", National Policies on Open Access (OA) Provision for University Research Output: an International meeting. Southampton UK: Southampton University. 
Carr, L. et al. 2006. Repositories for institutional open access: mandated deposit policies. Documento não publicado. Disponível em: https://core.ac.uk/download/files/34/1506254.pdf

Choo, C. W. 1998. Information management for the intelligent organization: the art of scanning the environment. 2 ed. Medford: ASIS/ Information Today.

Costa, S. 2006. "Filosofia aberta, modelos de negócios e agências de fomento: elementos essenciais a uma discussão sobre o Acesso Livre à informação científica”. Ciência da Informação 35 (2): 39-50.

Cresswell, J. W. 2010. Projeto de pesquisa: métodos qualitativo, quantitativo e misto. 3 ed. Porto Alegre: Artmed.

Crow, R. 2002. "The case for institutional repositories: A SPARC position paper". ARL Bimonthly Report 223.

Davenport, T. H. 1998. Ecologia da informação: por que só a tecnologia não basta para o sucesso na Era da Informação. São Paulo: Futura.

Declaração de Berlin. 2003. Conference on Open Access to Knowledge in the Sciences and Humanities. Berlin: Outubro.

Detlor, B. 2009. "Information management". International Journal of Information Management 30 (2): 103-108.

Diener, R. A. V. 1992. "Strategic, analytic and operational domains of information management". Bulletin of the American Society for Information Science 19 (1): 18-19.

Fairer-Wessels, F. A. 1997. "Information management education: towards a holistic perspective". South African Journal of Library and Information Science 65 (2): 93-102.

Fry, J. 2006. "Scholarly research and information practices: a domain analytic approach". Information Processing and Management 42 (1): 299-316.

Garvey, W. D. e B. C. Griffith. 1979. “Communication and information process within scientific disciplines, empirical findings for psychology”. W. D. Garvey, Communication: the essence of science: facilitating information among librarians, scientists, engineers and students. Oxford: Pergamon, 127-147.

Goffman, W. e K. S. Warren. 1980. Scientific information systems and the principle of selectivity. New York: Praeger.

Gorraiz, J. et al. 2009. "International publication output and research impact in social sciences: comparison of the Universities of Vienna, Zurich and Oslo". Research Evaluation 18 (3): 221-232.

Haridasan, S. e M. Khan. 2009. "Impact and use of e-resources by social scientists in National Social Science Documentation Centre (NASSDOC), India”. The Electronic Library 27 (1): 117-133.

Harnad, S. 2006. Optimizing OA self-archiving mandates: what? where? when? why? how? Technical Report, ECS, University of Southampton.

Harnad, S. e T. Brody. 2004. "Comparing the Impact of Open Access (OA) vs. Non- OA Articles in the Same Journals". D-Lib Magazine $10(6)$. 
Hills, P. J. 1983. “The scholarly communication process”. Annual Review of Information Science and Technology 8: 99-125.

Hine, C. M., ed. 2006. New infrastructure for knowledge production: understanding e-science. Hershey: Information Science Publishing.

Houghton, H. W., C. Steele e M. Henty. 2003. Changing research practices in the digital information and communication environment. Canberra: Department of Education, Science and Training.

Houghton, J. et al. 2009. Economic implications of alternative scholarly publishing models: exploring the costs and benefits. JISC EI-ASPM project. A report to the joint information systems committee (JISC). London: JISC.

Huang, M. e Y. Chang. 2008. "Characteristics of research output in social sciences and humanities: from a research evaluation perspective". Journal of the American Society for Information Science and Technology 59 (11): 1819-1828.

Hurd, J. M. 2004. "Scientific communication: new roles and new players”. Science \& Technology Libraries 25 (1): 5-22.

2000. "The transformation of scientific communication: a model for 2020". Journal of the American Society for Information Science 51 (14): 1279-1283.

1996. "Models of scientific communication systems". S. Y. Crawford, H. M. Hurd e A. C. Weller. From print to electronic: the transformation of scientific information. Medford: Asis, 9-33.

Jacobs, N., ed. 2006. Open access: key strategic, technical and economic aspects. Chandos Publishing: Oxford.

Jaeger, P. T. et al. 2005. "Information management". Encyclopedia of Social Measurement, v. 2.

Jamali, H. R. e D. Nicholas. 2008. "Information-seeking behavior of physicists and astronomers". Aslib Proceedings: New Information Perspectives 60 (5): 444-462.

Katz, J. S. e B. R. Martin. 1997. "What is research collaboration?" Research Policy 26.

Lagoze, C. e H. Van de Sompel. 2001. "The open archives initiative: building a low-barrier interoperability framework". ACM/IEEE Joint Conference on Digital Libraries, Roanoke, Virginia, 54-62.

Lancaster, F. W. e L. C. Smith. 1978. "Science, scholarship and the communication of knowledge". Library Trends 27 (3): 367-387.

Leite, F. C. L. 2011. Modelo genérico de gestão da informação científica para instituições de pesquisa na perspectiva da comunicação científca e do acesso aberto. Tese (Doutorado em Ciência da Informação), Brasília: Universidade de Brasília.

Lynch, C. A. 2003. "Institutional repositories: essential infrastructure for scholarship in the digital age". ARL Bimonthly Report 26.

Maron, N. L. e K. K. Smith. 2008 Current models of digital scholarly communication. Washington, DC: Association of Research Libraries. 
Meadows, A. J. 1999. A comunicação cientifica. Brasília: Briquet de Lemos.

Middleton, M. R. 2002. Information management: a consolidation of operations analysis and strategy. WaggaWagga: Charles Sturt University.

Mikhailov, A. I. et al. 1984. Scientific communications and informatics. Arlington: Information Resources.

Moore, G. 2011. Survey of University of Toronto Faculty Awareness, Attitudes, and Practices Regarding Scholarly Communication: A Preliminary Report. Toronto: University of Toronto.

Odlyzko, A. 2006. "Economic costs of toll access". N. Jacobs, ed. Open access: key strategic, technical and economic aspects. Oxford: Chandos Publishing.

Olson, G. M. et al., ed. 2008. Scientific colaboration on the internet. Cambridge: MIT Press.

Roosendaal, H. e P. Geurts. 1997. "Forces and functions in scientific communication: an analysis of their interplay". Cooperative Research Information Systems in Physics, August 31-September 4, Oldenburg, Germany.

Rowley, J. 1998. "Towards a framework for information management”. International Journal of Information Management 8 (5): 359-369.

Shearer, K. e B. Birdsall. 2002. The Transition of Scholarly Communication in Canada. CARL/ABRC Backgrounder. Ottawa: CARL/ABRC.

Søndergaard, T. F. et al. 2003. "Documents and the communication of scientific and scholarly information: revising and updating the UNISIST model". Journal of Documentation 59 (3): 278-320.

Suber, P. 2010. Open access overview: focusing on open access to peer-reviewed research articles and their preprints. Disponível em: http://legacy.earlham.edu/ peters/fos/overview.htm

— 2007. Timeline of the Open Access Movement. Disponivel em: http://legacy.earlham.edu/ peters/fos/timeline.htm

Swan, A. 2004. Journal authors survey: report. Cornwall: Key Perspectives.

2008. Key concerns within the scholarly communication process: report to the JISC Scholarly Communications Group. Truro: Key Perspectives Ltd.

_ 2006. "The culture of open access: researchers' views and responses". N. Jacobs, ed. Open access: Key strategic, technical and economic aspects. Oxford: Chandos.

- 2010. The open access citation advantage: studies and results to date. Technical Report. Southampton: University of Southampton.

Swan, A. e S. Brown. 2004. "Authors and open access publishing". Learned Publishing 17: 219-224.

2005. Open access self-archiving: an author study. Cornwall: Key Perspectives. 
UNISIST. 1971. Study report on the feasibility of a world science information system. Paris: Unesco.

Vickers, P. 1985. "Information management: selling a concept". B. Cronin, Information management: from strategies to action. Londres: Aslib, 151-160.

White, M. 1985. "Intelligence management". B. Cronin, ed. Information management: from strategies to action. Londres: Aslib, 21-35.

Willinsky, J. 2006. The access principle: the case for open access to research and scholarship. Massachusetts: MIT Press.

Wilson, T. D. 2002. "Information management". J. Feather e P. Sturges, ed. International Encyclopedia of Information and Library Science. Londres: Routleg.

Para citar este artículo:

Lima Leite, Fernando César y Sely Maria de Souza Costa. 2016. "Modelo genérico de gestão da informação científica para instituições de pesquisa na perspectiva da comunicação científica e do acesso aberto." Investigación Bibliotecológica: Archivonomía, Bibliotecología e Información 69: 43-73. http://dx.doi.org/10.1016/j.ibbai.2016.04.012 
